\title{
Iron Abundance in the Solar Photosphere: The Role of lons, Non-LTE, Model Atmospheres, Oscillator Strengths, and Free Parameters
}

Floe Foxon ( $\nabla$ floefoxon@protonmail.com )

Pinney Associates Inc https://orcid.org/0000-0002-4893-9178

\section{Research Article}

Keywords: Solar Photosphere, NLTE, Model Atmosphere, Oscillator Strength, Iron, Abundance

Posted Date: June 13th, 2022

DOI: https://doi.org/10.21203/rs.3.rs-1319069/v3

License: (1) (1) This work is licensed under a Creative Commons Attribution 4.0 International License.

Read Full License 


\title{
IRON ABUNDANCE IN THE SOLAR PHOTOSPHERE: THE ROLE OF IONS, NON-LTE, MODEL ATMOSPHERES, OSCILLATOR STRENGTHS, AND FREE PARAMETERS
}

\author{
FLOE FOXON \\ Version June 11, 2022
}

\begin{abstract}
The photospheric iron abundance is important to understanding solar system formation and evolution, but methodological designs in the literature have produced significantly different abundance estimates. 322 original photospheric iron abundances were extracted from 72 primary publications identified through SAO/NASA Astrophysics Data System and Google Scholar. Methodological characteristics corresponding to each abundance estimate were categorised, including the ion studied, local thermodynamic equilibrium (LTE) assumption, the atmospheric model and its dimensions, the source of oscillator strengths, and microturbulence velocity. A linear mixed-effects regression model was implemented to quantify the role these variables play in abundance estimates. The mean photospheric iron abundance across all sources was $7.44 \pm 0.02$ dex on the astronomical log scale. On the average, abundance estimates from Fe II lines were $0.106_{-0.061}^{+0.061}$ dex higher than those from Fe I lines (main effect), after controlling for other factors. Compared to LTE estimates, NLTE estimates were higher by $0.096_{-0.074}^{+0.073}$ dex (main effect). The role of LTE was different for neutral and ionised iron analyses. LTE abundance predictions were lower than NLTE predictions for Fe I, but higher than NLTE predictions for Fe II. Other differences are described for various model atmospheres and oscillator strengths. A subgroup analysis of $2641 \mathrm{D}$ and $\langle 3 \mathrm{D}\rangle$ model abundances suggests every $0.1 \mathrm{~km} \mathrm{~s}^{-1}$ increase in the microturbulence velocity parameter results in a 0.04 dex decrease in estimated abundance.
\end{abstract}

\section{INTRODUCTION}

In the late $19^{\text {th }}$ century, spectroscopic observations of absorption lines in the solar spectrum revealed the presence of 38 elements in the Sun (Rowland 1895). The first qualitative attempts to estimate the solar abundance of these elements were made by Russell (1914) and Moore \& Russell (1926). With the advent of ionization theory (Saha 1920 Saha \& Fowler 1921; Fowler \& Milne 1923), Payne (1925) produced the first quantitative estimates of the abundance of elements in the solar environment. Russell (1929) also produced new abundance estimates by calibrating Rowland intensities. However, the findings of Payne and Russell were limited in accuracy by numerous simplifying assumptions, and in the case of the latter, use of an arbitrary scale.

In the years that followed, the 'equivalent width' was developed as a metric for relating absorption line properties to abundances, first implemented by Minnaert \& Slob (1931). 'Curve of growth' methods with onedimensional model atmospheres and local thermodynamic equilibrium (LTE) became the dominant source of abundance estimates in the literature from the 1950s, until the 1990s when two- and three-dimensional models were introduced (Atroshchenko \& Gadun 1994, Gadun \& Pavlenko 1997). More recently, non-LTE (NLTE) abundance estimates have been published by Scott et al. (2015), Sitnova et al. (2015), Lind et al. (2017), Asplund et al. (2021), and Magg et al. (2022).

To date, dozens of articles on the photospheric iron abundance have been published, some controversially. Debate over the iron abundance was particularly intense in the 1990s with commentaries on articles (Holweger et al. 1995), commentaries on commentaries (Blackwell et al. 1995), and article titles such as 'The solar abundance of iron: a "final" word!' (Biemont et al. 1991) and 'The solar iron abundance: not the last word.' (Kostik et al. 1996). Much of this debate centred around the source of oscillator strengths used in abundance estimates, namely those measured by A. Bard, M. Kock, and co-authors at Kiel and Hannover, which resulted in 'low' iron abundances consistent with meteoritic iron, and those by D. E. Blackwell and co-authors at Oxford, which resulted in 'high' abundances $(\gtrsim 125 \%$ meteoritic). Grevesse \& Sauval (1999) suggest the cumulative effects of different choices for free parameters and oscillator strengths by these groups account for the high-low discrepancy. This raises the general question of what role oscillator strengths, free parameters, and other variables have played across the entire literature.

Understanding these aspects of abundance estimates is not immaterial. In general, accurate determinations of the relative elemental abundances are crucial to understanding solar system formation (McSween \& Huss 2010) and evolution (Kippenhahn et al. 2012). Outstanding problems involving the abundances include the First Ionization Potential (FIP) bias problem (Meyer 1985), which remains incompletely understood (Laming 2015. Prieto 2016), and discrepancies between observed primordial abundances and theoretical abundance predictions from Big Bang nucleosynthesis (Fields 2011). The abundance of iron is of particular importance because the iron abundance of a star is indicative of its overall metallicity (Asplund et al. 2009). Thus, better understanding of abundance methodology is necessary to answer open questions in astrochemistry.

The present study represents a secondary analysis of the photospheric iron abundance literature, which quantifies the impact of study design on abundance estimates. Specifically, statistical modelling is implemented to examine the roles of ions, LTE, model atmospheres, oscillator strengths, and microturbulence velocity. 


\section{METHODS}

\subsection{Data}

Data were collected from peer-reviewed publications returned from SAO/NASA Astrophysics Data System (ADS) and Google Scholar searches for 'solar iron abundance'. Additional articles were collected from references within the initial search results. Review articles which merely cited other abundance estimates and did not produce original abundance determinations, as well as proposed corrections for previously published abundance determinations, were excluded from analyses such that only primary sources were included. Additionally, the pioneering works of Payne (1925, with iron abundance 5.8 on the astronomical log scale), Russell (1929, 7.7), Menzel (Goldberg \& Aller 1943, 6.99), Unsöld (1945, 7.97) and Unsöld (1948, 7.26) were excluded because their methods differ so substantially from the remaining literature that comparisons are not informative.

Photospheric iron abundances on the astronomical log scale with Hydrogen abundance $A_{12}(H)=12$ were extracted from the identified publications, and the corresponding methodological characteristics from each study were categorised as follows.

1. The particular iron ion used was categorised as neutral iron (Fe I), singly ionised iron (Fe II), or both (Fe I \& Fe II).

2. Abundance estimates which assumed local thermodynamic equilibrium, that the electron temperature and excitation temperature are equal (Müller 1966), were categorised as LTE. Those that did not were categorised as non-LTE (NLTE).

3. The dimensions of the model atmosphere were categorised as one-dimensional (1D), two-dimensional (2D), three-dimensional (3D), or mean threedimensional $(\langle 3 \mathrm{D}\rangle)$, where the latter refers to $3 \mathrm{D}$ models averaged spatially (over horizontal layers/surfaces) and temporally.

4. The model atmospheres of Holweger (1967), Holweger \& Müller (1974), and modifications were categorised as HM. 'The atmospheres of Edvardsson et al. (1993), Asplund et al. (1997), and Gustafsson et al. (2008) were categorised as MARCS. The atmospheres of Fuhrmann et al. (1997), Grupp (2004), and Grupp et al. (2009) were categorised as MAFAGS. The Harvard-Smithsonian Reference Atmosphere of Gingerich et al. (1971), as well as Lites (1972) and Lites (1973), were categorised as HSRA. The atmospheres of Kurucz (1970), Kurucz (1992), and Kurucz (1993) were categorised as A'LAS. All other model atmospheres were categorised as 'Other.'

5. Oscillator strengths published by authors at Oxford (Blackwell et al. (1975), Blackwell et al. (1976), Andrews et al. (1979), Blackwell et al. (1979), Blackwell \& Shallis (1979), Blackwell et al. (1980), Blackwell et al. (1982a), Blackwell et al. (1982b), Blackwell et al. (1984), Blackwell et al. (1986), and Blackwell et al. (1995)) and Kiev (Gurtovenko \& Kostik (1981) and Gurtovenko \&
Kostik (1989)) were categorised as Oxford/Kiev. Oscillator strengths published by authors at Kiel and Hannover (Garz \& Kock (1969), Kock et al. (1984), Bard (1989), Heise \& Kock (1990), Bard et al. (1991), Bard \& Kock (1994), Schnabel et al. $(\overline{1999})$, Schnabel et al. (2004)) were categorised as Kiel/Hannover. Oscillator strengths published by C.H. Corliss and co-authors (Corliss \& Bozman (1962), Corliss \& Warner (1964), and Corliss \& Tech (1968)) were categorised as Corliss. The oscillator strengths of Meléndez \& Barbuy (2009) were categorised as Meléndez and Barbuy. All other oscillator strength sources, including combinations of the above categories, were categorised as 'Other.'

6. For $1 \mathrm{D}$ and $\langle 3 \mathrm{D}\rangle$ models, the microturbulence velocity parameter was recorded as a continuous variable in units of $\mathrm{km} \mathrm{s}^{-1}$.

The above categories were not selected arbitrarily. Rather, they represent the groupings of data with the largest number of abundance estimates. Because there are many obscure model atmospheres and oscillator strengths with few abundance determinations, the 'Other' categories are designed to prevent over-fitting from multi-level categorical variables (Babyak 2004). Green (1991) suggests a maximum number of predictors in multiple regression of $m=\frac{N-50}{8}$, where $N$ is the sample size (in this case, the number of abundance determinations lifted from the literature). Later in 3 it is shown that the sample size of the present study is sufficient for the above categories according to Green's rule.

\subsection{Model}

Data were analysed with a linear mixed-effects regression model of the form

$$
\mathbf{A}=\mathbf{X} \boldsymbol{\beta}+\mathbf{Z u}+\boldsymbol{\epsilon}
$$

where $\mathbf{A}$ is the column vector of photospheric iron abundance estimates from the literature, $\mathbf{X}$ is the design matrix of predictors (variables $1-5$ of 2.1 , , $\boldsymbol{\beta}$ is the column vector of fixed-effect regression coefficients, $\mathbf{Z}$ is the design matrix of random effects, which are the publications from which abundance estimates are taken, $\mathbf{u}$ is the column vector of random effect coefficients, and $\boldsymbol{\epsilon}$ is the column vector of the residuals. The random effects account for some confounding not controlled by the predictors. The categorical variables (1-5) are treated with dummy/indicator variables in the model, and an additional interaction term was included to quantify the relationship between neutral and ionised iron, and LTE. In the primary model with all abundance estimates, the microturbulence velocity variable was not included because 2D and 3D models do not have this parameter. However, a subgroup analysis of just $1 \mathrm{D}$ and $\langle 3 \mathrm{D}\rangle$ abundances was conducted with all variables including microturbulence. To be clear, this is not a model which relates iron abundance to year of publication, but one which relates iron abundance to the methods used to derive each iron abundance estimate in each publication (variables $1-6$ of 2.1 .

No attempt is made to weight data in the model by the uncertainty or error quoted for each abundance estimate. The is primarily because methods used to esti- 
mate uncertainty vary across the literature. Some studies present the standard deviation for the set of lines studied, some multiply this by two, and others estimate the standard error on the mean abundance (standard deviation divided by square root of the number of lines). These methods often provide overly optimistic performance assessments (as low as 0.002 dex!) Yet others provide more conservative error estimates not based on any of the above methods. Thus, weighting abundance estimates by quoted error would not be informative.

All analyses were conducted in Python version 3.8.8 with the packages numpy version 1.20 .1 , pandas version 1.2.4, matplotlib version 3.3.4, scipy version 1.6 .2 , and statsmodels version 0.12.2. All data and code prepared are available by request.

\section{RESULTS}

A total of 72 primary source articles with iron abundance determination were identified through ADS and Google Scholar searches and citations therein. The oldest of these is Claas (1951), and the most recent are Asplund et al. (2021) and Magg et al. (2022). 322 original abundance estimates were extracted from all articles total. According to Green's rule, this sample size allows for $m=\frac{322-50}{8}=34$ regression predictors. The number of predictors in the present model does not exceed Green's number.

The abundance estimates are plotted against publication year in Figure 1. Estimates have varied widely over time, concentrating in recent years between $A_{12}(F e)=$ 7.4 and 7.5 dex. Across all primary sources, the mean photospheric iron abundance \pm standard error is $7.44 \pm$ 0.02 dex.

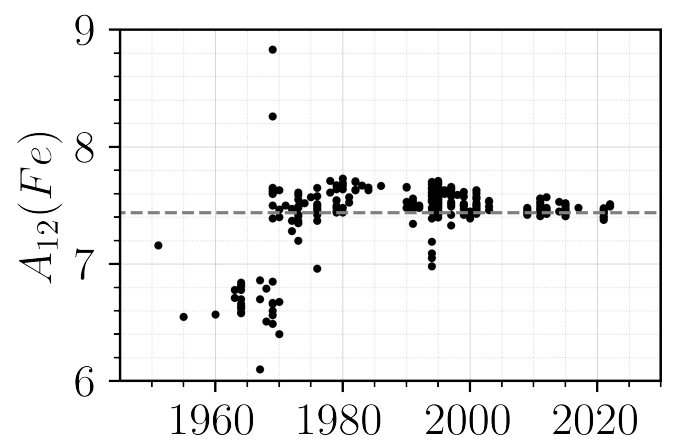

Figure 1. Iron abundance estimates from the primary literature plotted against year of publication. Abundances are expressed on the astronomical log scale on which the relative hydrogen abundance $A_{12}(H)=12$. The average iron abundance ( $\left.7.44 \mathrm{dex}\right)$ is shown by the dashed line.

Results from the primary regression model are found in Table 1. On the average, iron abundance estimates which analysed Fe II lines were significantly higher than those derived from Fe I lines by $0.106_{-0.061}^{+0.061}$ dex (main effect), after controlling for other factors. Although the coefficient for Fe I \& Fe II based abundances was not statistically significant, its value of 0.075 dex lies between zero and the Fe II coefficient of 0.106 dex, which is logical. Compared to LTE estimates, NLTE estimates were significantly higher by $0.096_{-0.074}^{+0.073} \mathrm{dex}$ (main effect). The interaction term between ion and thermodynamic assump- tion was also significant. The combined effect of ion and LTE on abundance is most intuitively quantified through out-of-sample predictions from the model. For two hypothetical abundances both derived from Fe I lines with the 1D HM model atmosphere and the Kiel/Hannover oscillator strengths, the LTE abundance prediction is lower than the NLTE prediction by approximately 0.1 dex. For the same model and oscillator strengths now with abundances derived from Fe II lines, the LTE abundance is higher than the NLTE prediction by approximately 0.05 dex. These findings suggest a significant difference in the role of LTE for neutral and ionised iron.

TABLE 1

Model Results

\begin{tabular}{|c|c|}
\hline Variable & Regression Coefficient (95\% CI) \\
\hline Constant & $7.480(7.382-7.578)^{* *}$ \\
\hline \multicolumn{2}{|l|}{ Ion } \\
\hline Fe I & 0.000 (Referent) \\
\hline Fe I \& II & $0.075(-0.045-0.195)$ \\
\hline Fe II & $0.106(0.045-0.167)^{* *}$ \\
\hline \multicolumn{2}{|l|}{ Thermodynamics } \\
\hline LTE & 0.000 (Referent) \\
\hline NLTE & $0.096(0.022-0.169)^{*}$ \\
\hline Ion $\times$ Thermodynamics & $(\text { See text })^{*}$ \\
\hline \multicolumn{2}{|l|}{ Model Dimensions } \\
\hline $1 \mathrm{D}$ & 0.000 (Referent) \\
\hline $2 \mathrm{D}$ & $-0.003(-0.175-0.169)$ \\
\hline $3 \mathrm{D}$ & $0.071(-0.018-0.159)$ \\
\hline$\langle 3 \mathrm{D}\rangle$ & $0.066(-0.025-0.156)$ \\
\hline \multicolumn{2}{|l|}{ Model Atmosphere } \\
\hline HM & 0.000 (Referent) \\
\hline ATLAS & $-0.055(-0.141-0.030)$ \\
\hline HSRA & $-0.070(-0.184-0.043)$ \\
\hline MAFAGS & $-0.052(-0.190-0.087)$ \\
\hline MARCS & $-0.087(-0.157--0.017)^{*}$ \\
\hline Other & $-0.122(-0.185--0.059)^{* *}$ \\
\hline \multicolumn{2}{|l|}{ Oscillator Strengths } \\
\hline Oxford/Kiev & 0.000 (Referent) \\
\hline Kiel/Hannover & $-0.066(-0.178-0.046)$ \\
\hline Corliss & $-0.540(-0.681--0.399)^{* *}$ \\
\hline Meléndez \& Barbuy & $-0.087(-0.217-0.043)$ \\
\hline Other & $-0.071(-0.160-0.018)$ \\
\hline
\end{tabular}

Surprisingly, differences in abundance estimates between 1D and 3D model atmospheres were, on the average, not statistically significant after controlling for other variables. Compared to HM-derived abundances, those derived from the ATLAS, HSRA, and MAFAGS model atmospheres were also not significantly different, though abundances with MARCS and Other models were significantly lower by $0.087_{-0.070}^{+0.070}$ dex and $0.122_{-0.063}^{+0.063}$ dex respectively. Finally, abundances from Kiel/Hannover oscillator strengths were lower than those from Oxford/Kiev oscillator strengths by $0.066 \mathrm{dex}$, however the effect did not reach statistical significance in this model. Corliss oscillator strengths did result in significantly lower abundances than Oxford/Kiev values.

In the subgroup analysis of $2641 \mathrm{D}$ and $\langle 3 \mathrm{D}\rangle$ abun- 
dances, the microturbulence velocity coefficient was $-0.407_{-0.066}^{+0.067}$ dex with $p<0.001$, implying that every $0.1 \mathrm{~km} \mathrm{~s}^{-1}$ increase in the microturbulence velocity parameter results in a significant decrease in the abundance estimate by approximately 0.04 dex.

An assessment of the performance of the model follows. Figure 2 shows model diagnostics by way of residuals, those being the differences between actual abundance estimates and the model's predictions. Figure $2 \mathrm{~A}$ visualises the actual and predicted abundances, showing that for most estimates predictions were accurate. The least accurate predictions were for the very lowest and the two very highest abundance estimates. The histogram in Figure $2 \mathrm{~B}$ suggests the residuals are approximately normally distributed with a mean of zero, therefore the underlying model assumption of linearity is valid.
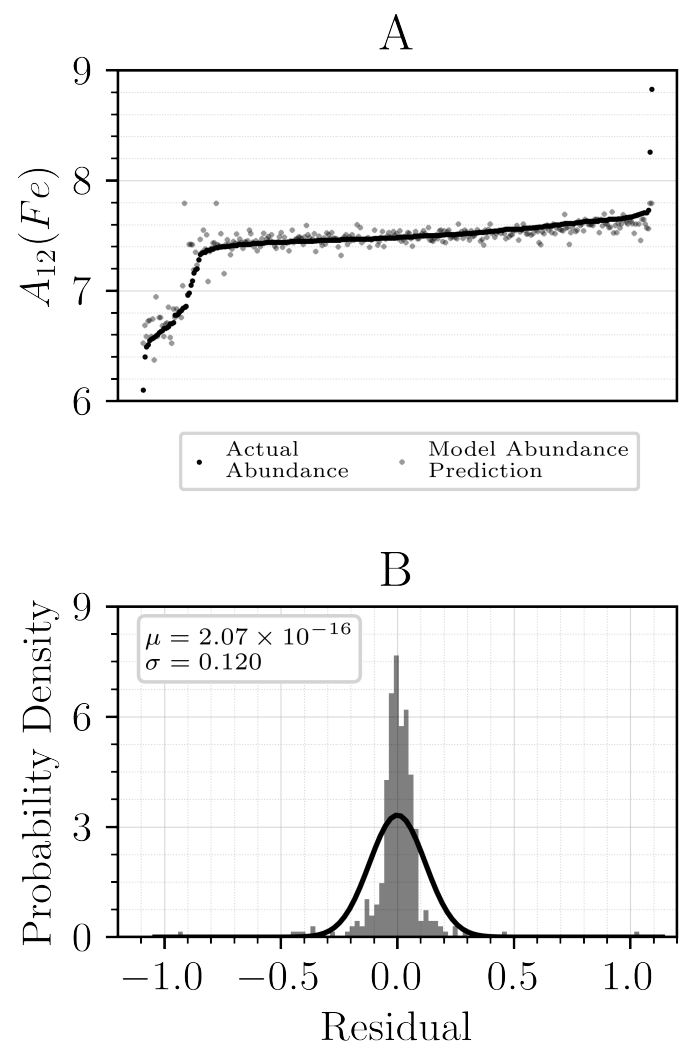

Figure 2. Model diagnostics. A: Actual iron abundance determinations from the primary literature plotted in ascending order with their corresponding model predictions. B: Histogram of residuals (difference between actual abundance from the primary literature and model abundance prediction). The number of bins follows the Freedman-Diaconis rule (Freedman \& Diaconis 1981).

\section{DISCUSSION}

In this study, an attempt was made to quantify the role of various methodological characteristics of iron abundance estimates in the published literature by way of a statistical model. The largest differences are accounted for by the ion studied and the LTE assumption. These two variables also interact such that the effect of LTE on the abundance estimate is opposite for neutral and ionised iron. While the oldest oscillator strengths from Corliss and co-authors made for significantly lower abundance estimates, differences were far less significant among more modern sources. The effect of choice of model atmosphere was also slight, however increasing values of the microturbulence velocity linearly reduced abundance estimates.

Several limitations should be noted, the first of which is that these findings do not indicate which study designs lead to 'correct' abundance estimates, but merely quantify the independent roles study variables play. Second, with limited sample size and low statistical power, non-significant $p$-values are to be expected. A significant $p$-value only indicates that an effect is non-zero, and a non-significant $p$-value is not proof that an effect is zero (Borenstein et al. 2011). This needs emphasising because the results of the present study do not imply that choices of model atmospheres and oscillator strengths have no effect on abundance estimates. Rather, the power of the present study was not sufficient to detect these effects of the order of 0.01 dex if they do exist. Third, the present results may be confounded by additional covariates which were not modelled, for example the wavelengths, excitation energies, equivalent widths, and $\log (g f)$ values corresponding to each line in each study. These are difficult to categorise because they are often not well reported. Rather than being a final 'final' word on the photospheric iron abundance, the present study serves as a reminder that careful consideration of study design is required in astrochemical abundance research. Researchers must be prepared to justify design decisions.

In their semi-recent compilation of solar photospheric abundances, Palme et al. (2014) recommend $A_{12}(F e)=$ $7.48 \pm 0.06$, though this was derived from the mean of a somewhat arbitrary selection of four abundance estimates with the same source of oscillator strengths. The uncertainty of 0.06 dex is a 'conservative' estimate. This result is reiterated in the most recent compilation by Lodders (2019), albeit with a less conservative uncertainty of 0.04 dex. The mean abundance across all sources found in the present study, $7.44 \pm 0.02$, is consistent with these recommendations, as well as the values most recently reported by Magg et al. (2022) (7.51 \pm 0.06$)$ and by Asplund et al. (2021) $(7.46 \pm 0.04)$, and with the iron abundance in CI-chondrites $(7.45 \pm 0.02)$ (Lodders 2019). 


\section{REFERENCES}

Andrews, J., Coates, P., Blackwell, D., Petford, A., \& Shallis, M. 1979, Monthly Notices of the Royal Astronomical Society, 186, 651

Asplund, M., Amarsi, A. M., \& Grevesse, N. 2021, A\&A, 653, A141

Asplund, M., Grevesse, N., Sauval, A. J., \& Scott, P. 2009, Annual review of astronomy and astrophysics, 47, 481

Asplund, M., Gustafsson, B., Kiselman, D., \& Eriksson, K. 1997, A\&A, 318, 521

Atroshchenko, I. N., \& Gadun, A. S. 1994, A\&A, 291, 635

Babyak, M. A. 2004, Psychosomatic medicine, 66, 411

Bard, A. 1989, PhD thesis, Institut für Plasmaphysik, Universität Hannover

Bard, A., Kock, A., \& Kock, M. 1991, Astronomy and Astrophysics, 248, 315

Bard, A., \& Kock, M. 1994, Astronomy and Astrophysics, 282, 1014

Bergemann, M., Lind, K., Collet, R., Magic, Z., \& Asplund, M. 2012, Monthly Notices of the Royal Astronomical Society, 427, 27

Biemont, E., Baudoux, M., Kurucz, R. L., Ansbacher, W., \& Pinnington, E. H. 1991, A\&A, 249, 539

Blackwell, D., Booth, A., Haddock, D., Petford, A., \& Leggett, S. 1986, Monthly Notices of the Royal Astronomical Society, 220, 549

Blackwell, D., Booth, A., \& Petford, A. 1984, Astronomy and Astrophysics, 132, 236

Blackwell, D., Ibbetson, P., \& Petford, A. 1975, Monthly Notices of the Royal Astronomical Society, 171, 195

Blackwell, D., Ibbetson, P., Petford, A., \& Willis, R. 1976, Monthly Notices of the Royal Astronomical Society, 177, 219

Blackwell, D., Lynas-Gray, A., \& Smith, G. 1995, Astronomy and Astrophysics, 296, 217

Blackwell, D., Petford, A., \& Shallis, M. 1979, Monthly Notices of the Royal Astronomical Society, 186, 657

Blackwell, D., Petford, A., Shallis, M., \& Simmons, G. 1980, Monthly Notices of the Royal Astronomical Society, 191, 445

-. 1982a, Monthly Notices of the Royal Astronomical Society, 199, 43

Blackwell, D., Petford, A., \& Simmons, G. 1982b, Monthly Notices of the Royal Astronomical Society, 201, 595

Blackwell, D., \& Shallis, M. 1979, Monthly Notices of the Royal Astronomical Society, 186, 669

Blackwell, D. E., Smith, G., \& Lynas-Gray, A. E. 1995, A\&A, 303, 575

Borenstein, M., Hedges, L., Higgins, J., \& Rothstein, H. 2011, Introduction to Meta-Analysis (Wiley), 11-14

Claas, W. J. 1951, The composition of the solar atmosphere.

Corliss, C. H., \& Bozman, W. R. 1962, Experimental Transition Probabilities for Spectral Lines of Seventy Elements Derived from the NBS Tables of Spectralline Intensities: The Wavelength, Energy Levels, Transition Probability, and Oscillator Strength of 25,000 Lines Between 2000 and 9000A for 112 Spectra of 70 Elements, Vol. 53 (US Government Printing Office)

Corliss, C. H., \& Tech, J. L. 1968, Oscillator strengths and transition probabilities for 3288 lines of Fe I, Vol. 108 (US Department of Commerce, National Bureau of Standards)

Corliss, C. H., \& Warner, B. 1964, The Astrophysical Journal Supplement Series, 8, 395

Edvardsson, B., Andersen, J., Gustafsson, B., et al. 1993, A\&A, 500,391

Fields, B. 2011, Annual review of nuclear science, 61, 47

Fowler, R. H., \& Milne, E. A. 1923, MNRAS, 83, 403

Freedman, D., \& Diaconis, P. 1981, Zeitschrift für Wahrscheinlichkeitstheorie und verwandte Gebiete, 57, 453

Fuhrmann, K., Pfeiffer, M., Frank, C., Reetz, J., \& Gehren, T. 1997, A\&A, 323, 909

Gadun, A. S., \& Pavlenko, Y. V. 1997, A\&A, 324, 281

Garz, T., \& Kock, M. 1969, Astronomy and Astrophysics, 2, 274

Gingerich, O., Noyes, R., Kalkofen, W., \& Cuny, Y. 1971, Solar

Physics, 18, 347
Goldberg, L., \& Aller, L. H. 1943, Atoms, stars and nebulae, 113-114

Green, S. B. 1991, Multivariate behavioral research, 26, 499

Grevesse, N., \& Sauval, A. 1999, Astronomy and Astrophysics, 347,348

Grupp, F. 2004, A\&A, 420, 289

Grupp, F., Kurucz, R. L., \& Tan, K. 2009, A\&A, 503, 177

Gurtovenko, E., \& Kostik, R. 1981, Astronomy and Astrophysics Supplement Series, 46, 239

-. 1989, Kiev, Naukova dumka, 1

Gustafsson, B., Edvardsson, B., Eriksson, K., et al. 2008, A\&A, 486,951

Heise, C., \& Kock, M. 1990, Astronomy and Astrophysics, 230, 244

Holweger, H. 1967, ZAp, 65, 365

Holweger, H., Kock, M., \& Bard, A. 1995, A\&A, 296, 233

Holweger, H., \& Müller, E. A. 1974, Sol. Phys., 39, 19

Kippenhahn, R., Weigert, A., \& Weiss, A. 2012, in Stellar Structure and Evolution (Springer), 73-81

Kock, M., Kroll, S., \& Schnehage, S. 1984, Physica Scripta, 1984, 84

Kostik, R. I., Shchukina, N. G., \& Rutten, R. J. 1996, A\&A, 305, 325

Kurucz, R. L. 1970, SAO Special Report, 309

-. 1992, Rev. Mexicana Astron. Astrofis., 23, 181

Kurucz, R.-L. 1993, Kurucz CD-Rom, 13

Laming, J. M. 2015, Living Reviews in Solar Physics, 12, 1

Lind, K., Amarsi, A. M., Asplund, M., et al. 2017, Monthly Notices of the Royal Astronomical Society, 468, 4311

Lites, B. 1972, University of Colorado and High Altitude Observatory, NCAR, Boulder

Lites, B. W. 1973, Solar Physics, 32, 283

Lodders, K. 2019, arXiv e-prints, arXiv:1912.00844

Magg, E., Bergemann, Maria, Serenelli, Aldo, et al. 2022, A\&A, 661, A140

McSween, H. Y., \& Huss, G. R. 2010, in Cosmochemistry (Cambridge University Press), 484-517

Meléndez, J., \& Barbuy, B. 2009, Astronomy \& Astrophysics, 497,611

Meyer, J. P. 1985, ApJS, 57, 173

Minnaert, M. G., \& Slob, C. 1931, Proc. Amsterdam Acad., 34, 542

Moore, C. E., \& Russell, H. N. 1926, ApJ, 63, 1

Müller, E. A. 1966, in Abundance Determinations in Stellar Spectra, ed. H. Hubenet, Vol. 26, 171

Palme, H., Lodders, K., \& Jones, A. 2014, Planets, Asteriods, Comets and The Solar System, Volume 2 of Treatise on Geochemistry (Second Edition). Edited by Andrew M. Davis. Elsevier, 2014., p. 15-36, 2

Payne, C. H. 1925, PhD thesis, Radcliffe College

Prieto, C. A. 2016, Living Reviews in Solar Physics, 13, 1

Rowland, H. A. 1895, Astrophysical Journal, 1, 29

Russell, H. N. 1914, Science, 39, 791

-. 1929, ApJ, 70, 11

Saha, M. N. 1920, The London, Edinburgh, and Dublin Philosophical Magazine and Journal of Science, 40, 472

Saha, M. N., \& Fowler, A. 1921, Proceedings of the Royal Society of London. Series A, Containing Papers of a Mathematical and Physical Character, 99, 135

Schnabel, R., Kock, M., \& Holweger, H. 1999, Astronomy and Astrophysics, 342, 610

Schnabel, R., Schultz-Johanning, M., \& Kock, M. 2004, Astronomy \& Astrophysics, 414, 1169

Scott, P., Asplund, M., Grevesse, N., Bergemann, M., \& Sauval, A. J. 2015, A\&A, 573, A26

Sitnova, T., Zhao, G., Mashonkina, L., et al. 2015, The Astrophysical Journal, 808, 148

Unsöld, A. 1945, Quantitative Spektralanalyse der

Sonnenatmosphäre (Vandenhoeck \& Ruprecht)

Unsöld, A. 1948, ZAp, 24, 306 